\title{
Feasibility of flotation concentration of fungal spores as a method to identify toxigenic mushrooms
}

This article was published in the following Dove Press journal:

Veterinary Medicine: Research and Reports

16 December 2014

Number of times this article has been viewed

Lisa J Bazzle'

Marc A Cubeta ${ }^{2}$

Steven L Marks'

David C Dorman ${ }^{3}$

'Department of Clinical Sciences, College of Veterinary Medicine,

${ }^{2}$ Department of Plant Pathology, College of Agriculture and Life

Sciences, Center for Integrated Fungal Research, ${ }^{3}$ Department of Molecular and Biomedical Sciences, College of Veterinary Medicine, North Carolina State University, Raleigh, NC, USA
Correspondence: David C Dorman Department of Molecular Biomedical Sciences, North Carolina State University, 1060 William Moore Drive, Raleigh, NC 27607, USA

$\mathrm{Tel}+19195136237$

$\mathrm{Fax}+19195136465$

Email david_dorman@ncsu.edu
Purpose: Mushroom poisoning is a recurring and challenging problem in veterinary medicine. Diagnosis of mushroom exposure in animals is hampered by the lack of rapid diagnostic tests. Our study evaluated the feasibility of using flotation concentration and microscopic evaluation of spores for mushroom identification. Evaluation of this method in living animals exposed to toxigenic mushrooms is limited by ethical constraints; therefore, we relied upon the use of an in vitro model that mimics the oral and gastric phases of digestion.

Methods: In our study, mycologist-identified toxigenic (poisonous) and nontoxigenic fresh mushrooms were collected in North Carolina, USA. In phase 1, quantitative spore recovery rates were determined following magnesium sulfate, modified Sheather's sugar solution, and zinc sulfate flotation ( $\mathrm{n}=16$ fungal species). In phase 2, mushrooms ( $\mathrm{n}=40$ fungal species) were macerated and digested for up to 2 hours in a salivary and gastric juice simulant. The partially digested material was acid neutralized, filtered, and spores concentrated using zinc sulfate flotation followed by microscopic evaluation of spore morphology.

Results: Mean spore recovery rates for the three flotation fluids ranged from $32.5 \%$ to $41.0 \%(P=0.82)$. Mean ( \pm standard error of the mean) Amanita spp. spore recovery rates were $38.1 \% \pm 3.4 \%, 36.9 \% \pm 8.6 \%$, and $74.5 \% \pm 1.6 \%(P=0.0012)$ for the magnesium sulfate, Sheather's sugar, and zinc sulfate solutions, respectively. Zinc sulfate flotation following in vitro acid digestion (phase 2) yielded spore numbers adequate for microscopic visualization in $97.5 \%$ of trials. The most common spore shapes observed were globose, spiked, elliptical, smooth and reticulate.

Conclusion: Flotation can concentrate mushroom spores; however, false negative results can occur. Spore morphology could not be used to differentiate species of mushroom-forming fungi since the spore shape and surface characteristics seen in the present study were often observed with multiple species of mushroom-forming fungi.

Keywords: gastrointestinal contents, mushroom spore identification, mushroom toxicity, Amanita spp.

\section{Introduction}

A mushroom is the aboveground spore-bearing fruiting body of a fungus. Mushrooms represent a wide variety of wild or cultivated gilled and poroid fungi with over 85 species identified as hazardous to human or animal health. ${ }^{1}$ Most poisonous species of mushrooms found in the United States can be characterized into eight broad categories based on the toxins that they produce: cyclopeptides (amatoxins), orellanine, monomethylhydrazine, coprine, ibotenic acid (muscimol), muscarine, psilocybin (psilocin), and gastrointestinal irritants. ${ }^{2}$ Toxic mushroom ingestion is associated with a variety of clinical signs in companion animals, with hepatic, neurologic, gastrointestinal, renal, and respiratory effects reported depending on the 
mushroom species ingested. ${ }^{3}$ Clinical prognosis following mushroom ingestion depends on the mushroom's toxicity, the amount ingested, and the animal's potential response to treatment. Timely gastrointestinal decontamination remains a hallmark of initial treatment of mushroom ingestion and depends upon the ability of veterinarians to rapidly diagnose mushroom exposure in their patients. ${ }^{3,4}$

Evidence of oral exposure to mushrooms can assume different forms. On occasion, people may witness the mushroom ingestion. At other times, pet owners may observe damaged mushrooms in the animal's environment, vomitus, or feces leading to an assumption that exposure occurred. Veterinarians managing these suspected exposures are often tasked with confirming exposure and developing a treatment plan that depends in part on their ability to identify the mushroom. In some cases, veterinarians will have access to specimens the client collected or material found in the animal's gastric contents. Identification of the mushroom species using these types of specimens can be challenging even for an experienced mycologist because morphologic variations can occur in the same mushroom genera and species depending on the season, geographic location, and maturity of the fungus. ${ }^{3,5}$ Evaluation of the shape and size of sexual spores (basidiospores) present in the ingested food or gastric contents has also been used to provide a tentative identification of mushroom-forming fungi in human clinical cases. ${ }^{6-11}$

Evaluation of food and gastric contents for the presence of mushroom spores can however be challenging. For example, low spore numbers found in feces may limit an individual's ability to detect spores during microscopic evaluation of gastric contents. Flotation concentration of spores in feces followed by microscopic evaluation may overcome this problem and has been used to confirm mushroom exposure in humans and animals. ${ }^{12-15}$ The clinical use of flotation concentration of mushroom spores, however, is largely limited to a few toxigenic mushroom species (eg, Amanita spp.), and its application has been confined to a few case reports. Clinical evaluation of spore flotation methods in veterinary medicine is hampered by the low incidence of mushroom poisoning cases in animals. Likewise, experimental studies using living animals are technically difficult, costly, and limited by ethical constraints when using potentially harmful mushrooms. Our study therefore relied upon the use of a two-step static in vitro model that mimics the oral and gastric phases of digestion as reproduced by a pepsin hydrolysis of mushrooms under fixed $\mathrm{pH}$ and temperature. Similar in vitro systems have been widely used in toxicology, microbiology, and food sciences. ${ }^{16-20}$
The primary objective of the present study was to evaluate whether mushroom spores could be concentrated with commonly used veterinary fecal flotation solutions. We hypothesized that flotation concentration would yield sufficient spore numbers for microscopic evaluation. Our study also provides a quantitative assessment of the effectiveness of flotation concentration of spores for a variety of mushroom species. In addition, we assessed spore morphology after mushroom digestion in gastric juice to determine whether microscopic spore features could be used to either identify or differentiate toxic and nontoxic mushrooms.

\section{Methods}

\section{Mushroom sample collection and identification}

The North Carolina State University Institutional Biosafety Committee approved all laboratory procedures. A variety of toxic and presumed nontoxic mushrooms ( $n=40$ samples) were collected within Wake County, NC, USA, between June 1 and October 20, 2013. Mushrooms were collected from a variety of habitats including grass lawns, pine forest, mixed hardwood forests, manure, and other locations. We sought specimens with minimal visual evidence of decay and favored collection of samples with multiple aboveground fruiting bodies. A professor of soil mycology and plant disease ecology (MAC) identified the genus and species of all mushrooms. Mushroom identification was based upon a combination of knowledge of the habitat, as well as macro- and microscopic morphologic characteristics of the mushrooms including cap color/shape, gill shape and degree of attachment to the stem (stipe), spore-bearing area (hymenium) associated with gills or pores, chemical reactions of spores and fruiting bodies, and the presence of a partial or universal veil, annulus (ring on the stem), or volva (Figure 1). Visual inspection of spore color and consistency of mushroom spore prints that were developed within 24 hours of mushroom collection on a colored paper substrate for each specimen were also used in the identification of mushroom samples.

\section{Flotation solutions and other reagents}

All purchased chemicals were reagent grade or higher (Sigma-Aldrich Company, St Louis, MO, USA). Saturated magnesium sulfate (450 $\mathrm{g} \mathrm{MgSO}_{4} / \mathrm{L} \mathrm{H}_{2} \mathrm{O}$ ), modified Sheather's sugar solution (454 g granulated sugar in $355 \mathrm{~mL}$ $\mathrm{H}_{2} \mathrm{O}$ with $6 \mathrm{~mL}$ formaldehyde), and 33\% zinc sulfate solutions (331 $\mathrm{g} \mathrm{ZnSO}_{4} / \mathrm{L} \mathrm{H}_{2} \mathrm{O}$ ) were used for spore flotation. The reported specific gravities for the magnesium sulfate, 


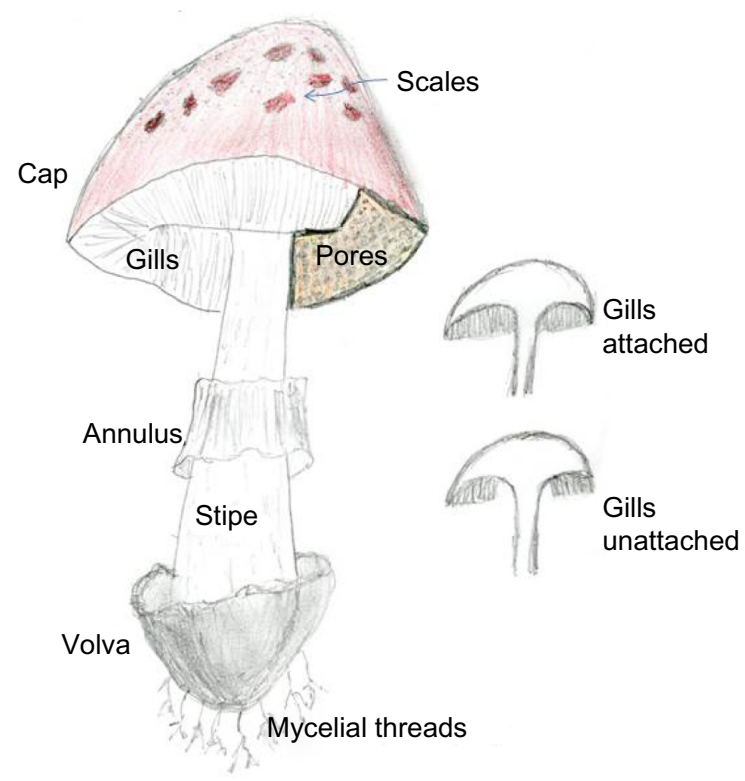

Figure I Macromorphological characteristics of mushrooms used for identification.

Sheather's sugar solution, and 33\% zinc sulfate solutions were $1.20,1.27$, and $1.18-1.20$, respectively. ${ }^{21}$

\section{Phase I: spore recovery rates with different flotation solutions}

This experimental phase used spore prints that were collected using a plastic petri dish as the substrate. After an overnight (12 hours) development of the spore print, the spores $(n=16$ mushroom samples) were scraped off the plastic substrate and suspended in $2 \mathrm{~mL}$ of distilled water and the initial spore concentration determined using a $10 \mu \mathrm{L}$ sample and a standard laboratory Neubauer hemocytometer. A $0.5 \mathrm{~mL}$ aliquot of the suspended spore sample was added to $1.5 \mathrm{~mL}$ of the magnesium sulfate, Sheather's sugar solution, or zinc sulfate solution in a $2 \mathrm{~mL}$ conical microcentrifuge tube for 60 minutes. Afterward, a laboratory pipette was used to slowly collect the top $(200 \mu \mathrm{L})$ portion (meniscus) of the sample. The final spore concentration was then determined using a $10 \mu \mathrm{L}$ sample and hemocytometer. Spore recovery rate (\%) was calculated from the ratio of the initial and final spore concentrations as adjusted for respective sample volumes.

\section{Phase 2: mushroom sample processing, in vitro digestion, and microscopic evaluation}

The mycologist-identified mushrooms ( $n=40$ samples) used in this experimental phase were digested using a modification of a previously described in vitro system that mimics gastrointestinal digestion. ${ }^{22}$ Briefly, a 5 to $10 \mathrm{~g}$ fresh sample of each mushroom specimen was chopped into 3 to $5 \mathrm{~mm}$ pieces to mimic maceration and incubated for approximately 1 to 1.5 minutes in $25 \mathrm{~mL}$ of a saliva fluid simulant. The saliva simulant fluid was prepared using an aqueous solution containing gastric mucin ( $1 \mathrm{~g} / \mathrm{L}), \alpha$-amylase $(2 \mathrm{~g} / \mathrm{L})$, sodium chloride $(0.117 \mathrm{~g} / \mathrm{L})$, potassium chloride $(0.149 \mathrm{~g} / \mathrm{L})$, and sodium bicarbonate $(2.1 \mathrm{~g} / \mathrm{L})$, with a final $\mathrm{pH}$ of 6.9 to 7.1 . Immediately afterward, the mushroom samples were transferred to $125 \mathrm{~mL}$ of an artificial aqueous gastric fluid solution, which was prepared with pepsin $(1 \mathrm{~g} / \mathrm{L})$, gastric mucin $(1.5 \mathrm{~g} / \mathrm{L})$, and sodium chloride $(8.775 \mathrm{~g} / \mathrm{L})$, with a final $\mathrm{pH} 1.8$ to 2.0 (adjusted using $1 \mathrm{~N}$ hydrochloric acid). The mushrooms and the gastric acid simulant were incubated at $37^{\circ} \mathrm{C}$ for 1.5 to 2 hours in a sealed, acid-resistant container in a standard cell culture incubator. All incubated samples were placed on an orbital rocker and gently agitated approximately three to five times per minute to mimic the frequency of normal canine stomach contractions. ${ }^{23}$ Following the incubation, the partially digested samples were neutralized (with $1 \mathrm{~N} \mathrm{NaOH}$ ) to approximately $\mathrm{pH} 6.5$ to 7.1 to match the $\mathrm{pH}$ of $\mathrm{dog}$ feces. ${ }^{24}$ Aliquots $(25 \mathrm{~mL})$ of the partially digested mushroom samples were removed at $0.5,1$, and 2 hours to evaluate the effect that varied gastric acid digestion times have on mushroom spore morphology. To mimic water absorption during feces formation, the partially digested mushroom samples were twice filtered using a Grade 5 cellulose filter paper (Whatman Inc., Florham Park, NJ, USA).

A $5 \mathrm{~g}$ aliquot of the filtered material was transferred to a $15 \mathrm{~mL}$ centrifuge tube for centrifugal flotation analysis. The 33\% zinc sulfate solution yielded the highest Amanita spore recovery rate in phase 1 (Figure 2) and this solution was subsequently used in phase 2 of this experiment. Thus, the $5 \mathrm{~mL}$ aliquot of the filtered material was initially well-mixed with $10 \mathrm{~mL}$ of the zinc sulfate solution, then additional zinc sulfate was added to the centrifuge tube to form a slight meniscus. Afterward a glass coverslip was placed over the meniscus for 1 hour to allow the spores to float to the top. The coverslip was subsequently transferred to a glass slide that was evaluated for spores using a light microscope equipped with $10 \times, 20 \times$, and $40 \times$ objectives (Olympus BX41, Olympus Corporation, Tokyo, Japan). Spore images were captured with a $40 \times$ objective using a digital camera (Cool SNAP, Roper Scientific Photometrics, Tucson, AZ, USA) equipped with RS Image (RS Image Software, Photometrics). All images were stored for future evaluation. A photographic atlas was created to illustrate the unique morphologic characteristics of common toxigenic and nontoxigenic mushroom spores. 


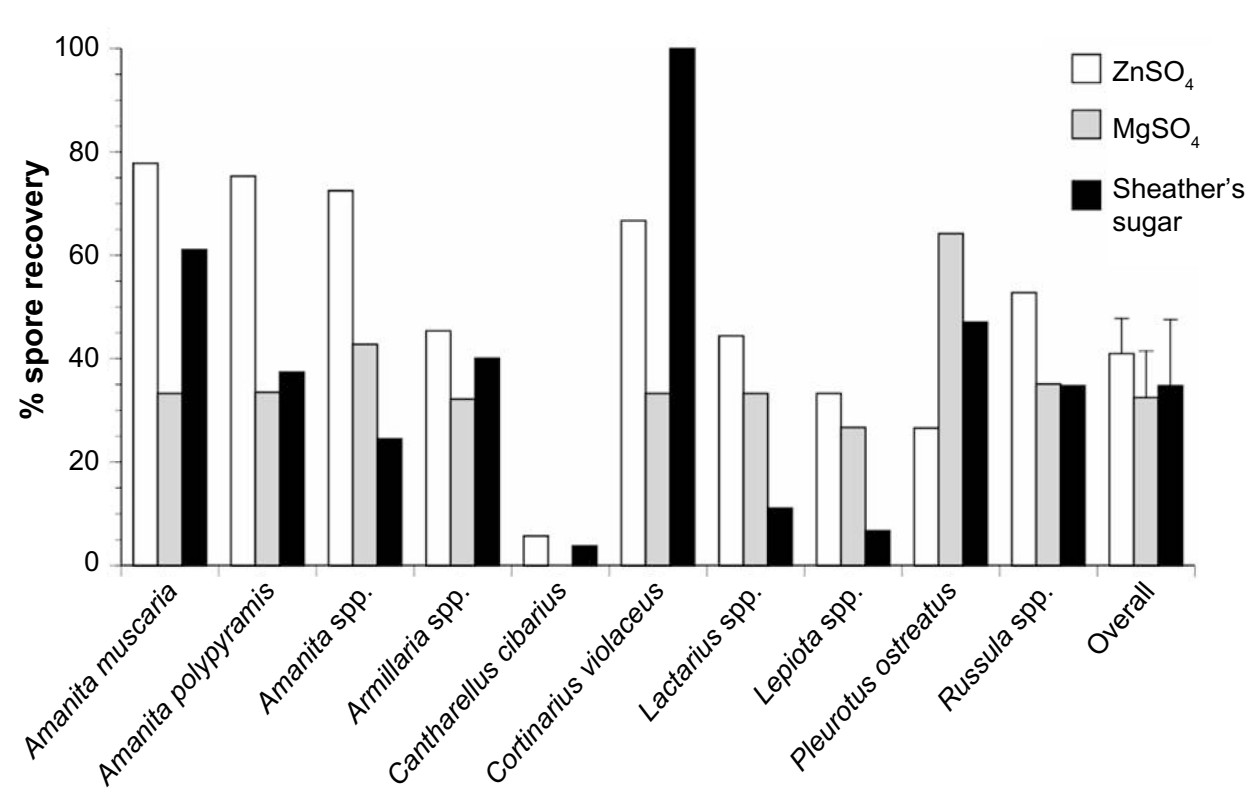

Figure 2 Spore recovery rates associated with zinc sulfate $\left(\mathrm{ZnSO}_{4}\right)$, magnesium sulfate $\left(\mathrm{MgSO}_{4}\right)$, and modified Sheather's sugar solution (phase I). Note: The overall ( $n=16$ samples) mean $( \pm S E M)$ spore recovery rates for the three flotation solutions are also shown.

Abbreviation: SEM, standard error of the mean.

\section{Statistical analysis}

Statistical analysis of spore recovery rates (phase 1) was performed following an assessment for homogeneity of variance (Levene's test) using an analysis of variance (ANOVA) and Dunnett's multiple comparison procedure for significant ANOVAs ( $F$-tests). All statistical analyses were performed using JMP Pro Version 9 (SAS Institute Inc., Cary, NC, USA). The results were considered statistically significant if $P \leq 0.05$. Unless otherwise reported all values represent mean \pm standard error of the mean (SEM) values.

\section{Results}

\section{Mushroom sample collection}

Forty mushrooms collected from Wake County, NC, USA, were identified to the level of either their genus or species. Mushrooms that could not be identified were excluded from further analysis (approximately $25 \%$ of collected mushroom samples). The 40 identified mushroom samples represented members of the following genera: Agaricus campestris $(\mathrm{n}=1)$, Amanita bisporigera $(\mathrm{n}=1)$, Amanita citrina $(\mathrm{n}=1)$, Amanita muscaria $(\mathrm{n}=1)$, and Amanita polypyramis $(\mathrm{n}=1)$, Amanita spp. $(\mathrm{n}=5)$, Armillaria mellea $(\mathrm{n}=3)$ and Armillaria tabescens $(\mathrm{n}=4)$, Boletus $\mathrm{spp} .(\mathrm{n}=2)$, Cantharellus cibarius $(\mathrm{n}=1)$, Cortinarius violaceus $(\mathrm{n}=2)$, Hygrocybe conica $(\mathrm{n}=1)$, Lactarius spp. $(\mathrm{n}=1)$, Leucoagaricus americanus $(\mathrm{n}=4)$, Leucocoprinus birnbaumii $(\mathrm{n}=1)$, Lycoperdon perlatum $(\mathrm{n}=2)$, Pleurotus ostreatus $(\mathrm{n}=1)$, Russula spp. $(\mathrm{n}=5)$, Scleroderma citrinum $(\mathrm{n}=2)$, and Suillus spp. $(\mathrm{n}=1)$. Known toxigenic mushrooms collected in this study included A. bisporigera, A. citrina, A. muscaria, A. polypyramis, L. birnbaumii, Lycoperdon spp. (toxic when inhaled), and S. citrinum (Table 1)

\section{Quantitative spore recovery rates with different flotation solutions}

Figure 2 shows the spore recovery rates for the ten species of mushroom-forming fungi used in phase 1 of the experiment. No statistically significant difference in spore recovery rate ( $\mathrm{n}=16$ mushroom samples) was observed with the three flotation fluids ( $P=0.82$; ANOVA). The overall ( $\mathrm{n}=16$ samples) mean $( \pm$ SEM) spore recovery rates for the three flotation fluids were $32.5 \% \pm 9.0 \%, 34.8 \% \pm 12.8 \%$, and $41.0 \% \pm 6.8 \%$ for the saturated magnesium sulfate, modified Sheather's sugar solution, and zinc sulfate solutions, respectively. Because of their clinical importance and known toxigenic properties we also assessed whether the different flotation solutions affected Amanita spore recovery rates. We observed a statistically significant difference ( $P=0.0012$, ANOVA) in Amanita spp. ( $\mathrm{n}=4$ mushroom samples) spore recovery rates. The mean ( \pm SEM) Amanita spore recovery rates for the three flotation fluids were $38.1 \% \pm 3.4 \%, 36.9 \% \pm 8.6 \%$, and $74.5 \% \pm 1.6 \%$ for the saturated magnesium sulfate, modified Sheather's sugar solution, and zinc sulfate solutions, respectively. We also evaluated the coefficient of variation when different flotation solutions were used with identical Amanita spp. samples run in triplicate. The observed coefficients of variation were 
Table I Spore shapes observed following in vitro gastric acid digestion and zinc sulfate flotation (phase 2)

\begin{tabular}{|c|c|c|c|c|}
\hline Mushroom species & Spore shape & Spore print color & Toxicity & Toxic mechanism \\
\hline Agaricus campestris & Elliptical $^{a}$ & Brown & Generally nontoxic $^{b}$ & Not applicable \\
\hline Amanita spp. & Globose & White & Not applicable & Not applicable \\
\hline $\begin{array}{l}\text { Amanita muscarial } \\
\text { Amanita polypyramis }\end{array}$ & Globose & White & Toxic & $\begin{array}{l}\text { Muscarine, muscimol, ibotenic acid, } \\
\text { cyclopeptides }\end{array}$ \\
\hline Armillaria mellea & Elliptical & White & Nontoxic & Not applicable \\
\hline Armillaria tabescens & Elliptical & White & Toxic when consumed raw & Gastrointestinal irritant \\
\hline Boletus spp. & Elliptical ${ }^{\mathrm{c}}$ & Olive-brown & Generally nontoxic ${ }^{\mathrm{b}}$ & $\begin{array}{l}\text { Muscarine-containing and/or } \\
\text { gastrointestinal irritants }\end{array}$ \\
\hline Cantharellus cibarius & Elliptical & White/yellow & Nontoxic & Not applicable \\
\hline Cortinarius violaceus & Elliptical/spiked ${ }^{d}$ & Brown & Generally nontoxic ${ }^{b}$ & Other species with orellanine \\
\hline Hygrocybe conica & Elliptical & White & Nontoxic & Not applicable \\
\hline Lactarius spp. & Elliptical/spiked ${ }^{d}$ & Beige/white & Generally nontoxic ${ }^{b}$ & Gastrointestinal irritant \\
\hline Leucoagaricus americanus & Elliptical & White & Possible & Not applicable \\
\hline Leucocoprinus birnbaumii & Elliptical $^{a}$ & White & Toxic & Gastrointestinal irritant \\
\hline Lycoperdon perlatum & Globose/spiked $^{d}$ & Olive-brown & Toxic if inhaled & Respiratory irritant \\
\hline Pleurotus ostreatus ${ }^{\mathrm{e}}$ & Elliptical & White & Nontoxic & Not applicable \\
\hline Russula spp. & Elliptical/spiked ${ }^{d}$ & White to orangish & Generally nontoxic ${ }^{b}$ & Gastrointestinal irritant \\
\hline Scleroderma citrinum & Globose & Purple-black & Toxic & Gastrointestinal irritant \\
\hline Suillus spp. & Spindle-shaped ${ }^{c}$ & Brown & Nontoxic & Not applicable \\
\hline
\end{tabular}

Notes: Spore shape nomenclature described by Hall et al, 2003. ${ }^{39}$ a Apical pore present; ${ }^{\text {bsome species are toxic; ' } g l o b u l e s ~ p r e s e n t ; ~}{ }^{\text {s}}$ spiked, warted (reticulate), not smooth; espores not recovered.

18.1, 46.8, and 4.4 for the saturated magnesium sulfate, modified Sheather's sugar solution, and zinc sulfate solutions, respectively.

\section{Qualitative spore recovery and morphology}

Of the 40 mushrooms used in phase 2 of the study, only one species (P. ostreatus) did not yield a sufficient number of spores to be seen microscopically. A variety of spore shapes were observed following acid digestion and flotation. The spore shapes were classified as either globose (Figure 3),

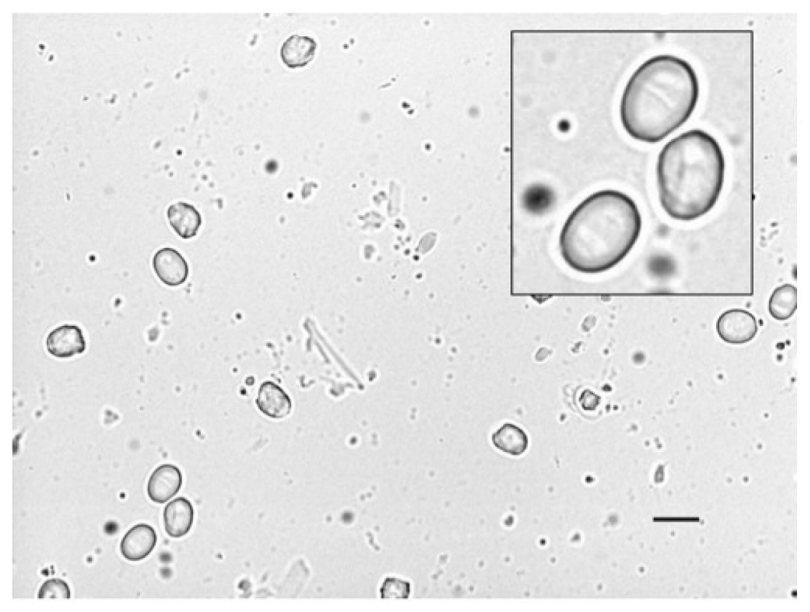

Figure 3 Globose spore shape seen following in vitro acid digestion and zinc sulfate flotation (phase 2).

Notes: This spore shape is associated with species of Amanita and Armillaria. Inset shows higher magnification of several spores. Scale bar $=7.5 \mu \mathrm{m}$. elliptical with or without apical pores or globules (Figure 4), spiked round or elliptical, or reticulate (Figure 5). Spore shapes and colors seen with the different mushroom species are summarized in Table 1. The most common spore shape was globose (41.0\%), followed by spiked (28.2\%), elliptical $(25.6 \%)$, and reticulate $(5.1 \%)$. There was no association between spore shape, color, and a mushroom's reported toxicity (Table 1). The duration of the acid digestion procedure (up to 2 hours) did not adversely affect spore morphology (data not shown).

\section{Discussion}

One of our first objectives was to identify the species of mushrooms collected for our study. Visual inspection of spore prints played an important role in the identification of the collected mushroom specimens. The color of the spore
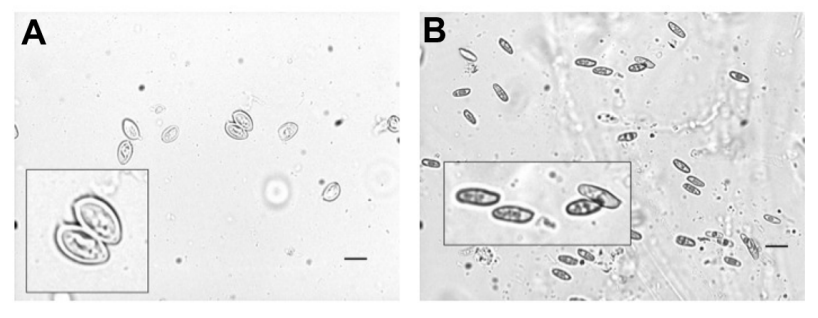

Figure 4 Microscopic spore shapes seen following in vitro acid digestion and zinc sulfate flotation.

Notes: (A) Elliptical without globules; this spore shape is associated with species of Agaricus campestris, Hygrocybe conica, Leucoagaricus americanus, and Leucocoprinus birnbaumii. (B) Elliptical with globules; these spores were associated with Boletus and Suillus species. Insets show higher magnification of spores. Scale bar $=7.5 \mu \mathrm{m}$. 


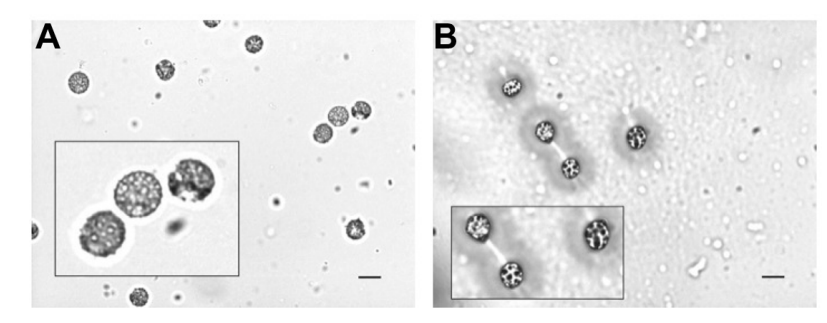

Figure 5 Representative microscopic spore shapes seen following in vitro acid digestion and zinc sulfate flotation.

Notes: (A) Spiked (reticulate) spore shape associated with species of Cortinarius violaceus, Lactarius, Lycoperdon perlatum, and Russula. (B) Reticulate spore shape associated with Scleroderma citrinum. Insets show higher magnification of several spores. Scale bar $=7.5 \mu \mathrm{m}$.

print obtained from the mushroom sample was compared with published descriptions available in field guides and keys. We found that interpreting spore color was at times subjective and standardized descriptors (eg, white versus creamy) were generally lacking in the published keys. Approximately $25 \%$ of all collected mushrooms did not develop a recognizable spore print and this finding likely reflected the age of the mushroom specimen (ie, too old or immature). Microscopic evaluation of the spores obtained from the print also revealed important information about spore morphology, size, and color that could further aid in identification. As expected, distinguishing white, brown, pink, red, black, or other spore print colors from one another remained a useful identification tool but could not be used in isolation to make a taxonomic determination of the mushroom specimen. Other gross characteristics were therefore needed to identify the mushroom specimens (Figure 1). Often the entire mushroom including the base was necessary to support identification of specific mushrooms. Despite considering these varied criteria, the identification of individual species of mushrooms remained challenging even with the aid of an experienced mycologist and was a primary limitation of this study. Indeed, an experienced mycologist could not definitively identify the genus/species level of approximately $25 \%$ of the mushroom specimens we collected in the present study.

Our study was prompted by our observation that a number of textbook and journal authors have suggested that microscopic evaluation of spores in gastrointestinal contents could be a viable adjunct to other diagnostic tests. ${ }^{12-15}$ In particular, we evaluated whether common flotation methods used in veterinary practice could be used to concentrate mushroom spores and improve microscopic evaluations. These flotation methods exploit a physical property (specific gravity) of spores and other materials whereby objects with a lower specific gravity will float in a denser solution. ${ }^{25}$ Veterinarians are well acquainted with fecal concentration methods that allow for the detection of small numbers of ova and parasites that may be missed by using only a direct wet smear. In veterinary parasitology, the flotation procedure permits the separation of protozoan cysts, coccidian oocysts, microsporidian spores, and certain helminth eggs and larvae with a specific gravity of 1.05 to 1.25 from the more dense $(>1.3)$ background fecal material through the use of a liquid with a high specific gravity. ${ }^{25-27}$ The less dense parasitic elements are recovered in the surface film, and the denser fecal debris remains in the bottom of the tube yielding a cleaner preparation that can be examined microscopically. ${ }^{25}$ We used a similar approach to concentrate the mushroom spores in our acid-digested materials. We found that for most mushrooms the type of flotation solution (eg, saturated magnesium sulfate, modified Sheather's sugar solution, and zinc sulfate solution) yielded qualitatively similar spore recovery rates with approximately $33 \%$ to $41 \%$ of the total spores found in the uppermost $10 \%$ volume of the sample. A statistically significant difference was noted with Amanita spp. spore recovery rates. In this case, the less dense zinc sulfate solution yielded an approximately twofold higher spore recovery rate (75\%) when compared with either the magnesium sulfate or Sheather's sugar solutions. We found that zinc sulfate flotation of partially digested mushroom samples yielded spore numbers adequate for microscopic visualization in $97.5 \%$ of trials where a spore print formed as part of our initial mushroom identification. Although not studied directly in our experiment, concentration of the spores by flotation will likely be superior to microscopic evaluation of direct smears of vomitus, feces, or other gastric contents. Likewise, since the specific gravities of the solutions we used were less dense than that reported for fecal debris, we anticipate that these methods will also allow adequate separation of the less dense mushroom spores studied in our experiment from fecal debris in veterinary clinical cases. This assumption is supported by clinical reports demonstrating positive mushroom spore identification from human and animal feces..$^{12-15}$

Despite our high success rate at concentrating spores with flotation, veterinarians must remain aware that false negative results may occur. For example, poor spore recovery following flotation could occur depending upon the age of the mushrooms (ie, immature or hypermature spores), excessive dilution/inadequate concentration during filtration, or spore density in excess of the flotation fluid density. Another clinically important factor that may contribute to false negative results is the long latent period between exposure and the onset of clinical signs seen with some mushrooms. For example, ingestion of hepatotoxic Amanita mushroom is often 
associated with an initial asymptomatic latent period that lasts 6-12 hours post-ingestion and a hepatotoxic phase that lasts an additional 24-36 hours. ${ }^{28,29}$ Negative fecal flotation results may occur clinically since the Amanita spores may have been eliminated prior to the collection of a fecal sample. When considered collectively, the absence of visualized spores in gastric/fecal contents would not be anticipated to rule out a potential ingestion of a toxic mushroom.

In our study, the mushroom spores obtained following flotation were then subjected to microscopic evaluation. Our initial hope was to develop a photographic key to assist in the identification of different mushroom species based upon spore shape, size, and other physical characteristics. We found, however, that spores from more than one mushroom species shared several shape/color/size characteristics suggesting that the microscopic appearance of the spore was best used to exclude certain mushroom species from consideration rather than to provide positive identification. Although spore morphology could help narrow the identification of a digested mushroom to one or more genera, these physical characteristics often could not narrow the identification any further. For example, four different species of Amanita were collected during the course of our study, all of which were microscopically similar in regard to spore shape following acid digestion and zinc sulfate flotation. When considered collectively, the mushrooms' macroscopic physical features or microscopic spore characteristics were sometimes insufficient to differentiate individual species within a mushroom genus. This may be clinically important for Amanita mushrooms and other genera that contain both toxigenic (poisonous) and nontoxigenic (nonpoisonous) species. We also observed that spore size could vary within a specimen further complicating interpretation of our results. Challenges associated with the use of spore size and morphology has been documented in the literature. For example, misidentifications of Cortinarius mushrooms based on spore morphology and size have been reported in the human clinical literature. ${ }^{8}$ Other studies have shown that spore size can vary depending upon season, time of day, and other factors. ${ }^{30}$

Veterinarians may need to rely upon additional diagnostic tests to confirm the species of mushroom involved. For example, molecular biology approaches, including the use of polymerase chain reaction analysis, are often required to definitively diagnose a specific Amanita (or other) mushroom species. ${ }^{31-35}$ Additional analytical chemical methods are also available to confirm certain mushroom exposures. For example, analytical approaches exist to detect amanitins (amatoxins) in bile and urine. ${ }^{3,35}$ The amanitins inhibit ribonucleic acid polymerase II and cause the acute hepatocellular necrosis and mortality associated with Amanita phalloides and other hepatotoxic Amanita species. ${ }^{36}$ These types of analyses were, however, considered beyond the scope of the present study.

One limitation of the present study was that the geographical area we used to collect mushrooms was only representative of one region (Wake County, NC, USA). Our search strategy for mushrooms considered the veterinary medical literature. For example, the period when mushroom samples were collected in our study coincided with when the greatest diversity and fruiting of mushroom-forming toxigenic and nontoxigenic species occur in this region. In addition, most published veterinary case reports of suspected mushroom intoxication indicate that exposure occurred in moist, cool areas during the fall months. ${ }^{3}$ We also considered local growing conditions and reported habitats for these mushrooms in designing our search strategy. For example, A. phalloides (also known as the death cap) is found along the west- and east-coast, growing commonly near pine, oak, and birch trees in the fall months. We had one study limitation imposed by our Institutional Biosafety Committee that precluded collection of Psilocybe mushrooms because of their status as a Schedule I substance under the US Controlled Substances Act. Despite this geographical constraint, sufficient data were obtained to identify some of the important strengths and limitations of using flotation concentration for mushroom spores.

An important strength of our study was the use of an in vitro digestion method. Because of the large number of samples collected $(n=40)$ and the inherent toxicity of some mushroom species, we elected to use an in vitro approach rather than rely on dosing of animals with poisonous mushrooms. Likewise, relying on spontaneous cases of mushroom exposures seen in the North Carolina State University Veterinary Health Complex would also be problematic because of the complex's low annual incidence rate of confirmed mushroom poisoning and the lack of definitive mushroom identification in most clinical cases. The in vitro approach we used was chosen to mimic the harshest digestion conditions that the mushroom samples would be exposed to following ingestion. As with any experimental model, the in vitro system we used has some limitations. For example, our system did not attempt to replicate conditions in the small and large intestines. Our study, therefore, cannot directly address whether bile and other gastrointestinal contents could affect spore morphology or size. However, the spore color and morphology seen in our study following acid digestion were consistent with 
our evaluation of spores obtained from spore prints and previously published results. ${ }^{37}$ In addition, published images of mushroom spores collected from people or animals with confirmed toxic mushroom exposure suggest that the presence of bile and other materials did not significantly alter the physical appearance of the spores. ${ }^{12,13}$ The methodological limitations associated with our in vitro system will likely not impact the application of our study results to the interpretation of spores present in vomitus or gastric contents. This is an important consideration since many mushroom-exposed animals are initially treated with emetics and vomitus samples may be available for analysis. ${ }^{3}$

\section{Conclusion}

Diagnosing mushroom exposure or poisoning remains a significant clinical challenge, in part because of the variable time course of clinical signs associated with different species of toxic mushrooms and the limited number of diagnostic aids available. ${ }^{38}$ Our study suggests that flotation concentration of mushroom spores is a feasible approach to concentrate mushroom spores. Further, we anticipate that the flotation methods used in our in vitro study could be easily applied to vomitus, feces, and other clinically relevant samples. Interpretation of negative clinical flotation results, however, will require caution since our study suggests that false negative results may occur. We have also shown that microscopic evaluation of concentrated spores may assist in the identification of mushrooms. Microscopic evaluation of spore morphology, however, may have its greatest clinical application in ruling out certain mushroom species. Because of the observed similarity in certain spore morphological characteristics and known variation in the spore characteristics based on the maturity of the spores, microscopic evaluation of spores is less likely to yield diagnostic information that will confirm the identity of a mushroom. In these cases, additional methods including physical characteristics of the fruiting body and chemical and/or molecular analyses of mushroom or tissue specimens may be needed to reach a definitive taxonomic conclusion. Because of these difficulties in identifying mushrooms, confirming exposure, and the lack of effective antidotes for many mushrooms, initial management approaches should remain focused on client education to prevent exposure and timely gastrointestinal decontamination in all cases in which mushroom exposure was suspected.

\section{Acknowledgments}

This research was supported in part through an unrestricted gift from Afton Chemical Corporation. The authors thank
Drs Michael Stoskopf and Susan Kennedy-Stoskopf for access to their farm for mushroom collection. We also thank Kris Alpi and Dr Wolfgang Baeumer for their assistance with article translation.

\section{Disclosure}

The authors report no conflicts of interest in this work.

\section{References}

1. Ford M, Delaney KA, Ling LJ, Erickson T, editors. Clinical Toxicology. 1st ed. Saint Louis: WB Saunders; 2000.

2. Rumack BH, Spoerke DG, editors. Handbook of Mushroom Poisoning: Diagnosis and Treatment. 1st ed. Boca Raton: CRC Press; 1994.

3. Puschner B, Wegenast C. Mushroom poisoning cases in dogs and cats: diagnosis and treatment of hepatotoxic, neurotoxic, gastroenterotoxic, nephrotoxic, and muscarinic mushrooms. Vet Clin North Am Small Anim Pract. 2012;42(2):375-387.

4. Spoerke D. Mushrooms. In: Peterson ME, Talcott PA, editors. Small Animal Toxicology. 2nd ed. Saint Louis: WB Saunders; 2006:860-887.

5. Petersen RH, Hughes KW. Species and speciation in mushrooms? Development of a species concept poses difficulties. Bioscience. 1999;49(6):440-452.

6. Apperley S, Kroeger P, Kirchmair M, Kiaii M, Holmes DT, Garber I. Laboratory confirmation of Amanita smithiana mushroom poisoning. Clin Toxicol (Phila). 2013;51(4):249-251.

7. Stř́ibrný J, Sokol M, Merová B, Ondra P. GC/MS determination of ibotenic acid and muscimol in the urine of patients intoxicated with Amanita pantherina. Int J Legal Med. 2012;126(4):519-524.

8. Kirchmair M, Pöder R. Fatal renal failure caused by Cortinarius mushrooms. Pediatr Nephrol. 2011;26(3):487-488.

9. Frank H, Zilker T, Kirchmair M, et al. Acute renal failure by ingestion of Cortinarius species confounded with psychoactive mushrooms: a case series and literature survey. Clin Nephrol. 2009;71(5):557-562.

10. Horn S, Horina JH, Krejs GJ, Holzer H, Ratschek M. End-stage renal failure from mushroom poisoning with Cortinarius orellanus: report of four cases and review of the literature. Am J Kidney Dis. 1997;30(2): 282-286.

11. Pohle W, Wöllner-Siebert G. [Spore analysis, an important aid in the diagnosis of mushroom poisoning]. Z Arztl Fortbild (Jena). 1983;77(4):144-158. [German].

12. Machbert G, Wiesmeier I. [Diagnosis of mushroom poisoning by concentration of spores found in gastrointestinal contents and feces]. Z Rechtsmed. 1971;68(3):115-123. [German].

13. Tomini L, Gregorutti S, Triolo L. [Sporological diagnosis in 282 cases of suspected fungal poisoning occurring in the municipality of Trieste from 1967 to 1984]. Minerva Med. 1987;78(5):321-327. [Italian].

14. Maser C, Maser Z. Notes on mycophagy in four species of mice in the genus Peromyscus. Great Basin Nat. 1987;47(2):308-313.

15. Rafferty B, Dowding P, McGee EJ. Fungal spores in faeces as evidence of fungus ingestion by sheep. Sci Total Environ. 1994;157(1-3): $317-321$.

16. Guerra A, Etienne-Mesmin L, Livrelli V, Denis S, Blanquet-Diot S, Alric M. Relevance and challenges in modeling human gastric and small intestinal digestion. Trends Biotechnol. 2012; 30(11):591-600.

17. Hu JL, Nie SP, Min FF, Xie MY. Artificial simulated saliva, gastric and intestinal digestion of polysaccharide from the seeds of Plantago asiatica L. Carbohydr Polym. 2013;92(2):1143-1150.

18. Li PK, Spittler C, Taylor CW 3rd, Sponseller D, Chung RS. In vitro effects of simulated gastric juice on swallowed metal objects: implications for practical management. Gastrointest Endosc. 1997; 46(2):152-155.

19. Rebhandl W, Milassin A, Brunner L, et al. In vitro study of ingested coins: leave them or retrieve them? Pediatr Surg. 2007;42(10):1729-1734. 
20. Rebhandl W, Steffan I, Schramel P, et al. Release of toxic metals from button batteries retained in the stomach: An in vitro study. J Pediatr Surg. 2002;37(1):87-92.

21. Dryden MW, Payne PA, Ridley RK, Smith VE. Gastrointestinal parasites: The practice guide to accurate diagnosis and treatment. Compend Cont Educ Vet. 2006;28(8A):3-14.

22. Kong F, Singh RP. A model stomach system to investigate disintegration kinetics of solid foods during gastric digestion. J Food Sci. 2008;73(5):E202-E210.

23. Hall JE. Guyton and Hall Textbook of Medical Physiology. 12th ed. Philadelphia: Saunders; 2011.

24. Blagburn BL, Butler JM. Optimize intestinal parasite detection with centrifugal fecal flotation. Vet Med. 2006;101(7):455-464.

25. Brambillasca S, Purtscher F, Britos A, Repetto JL, Cajarville C. Digestibility, fecal characteristics, and plasma glucose and urea in dogs fed a commercial dog food once or three times daily. Can Vet J. 2010;51(2):190-194.

26. Foreyt WJ. Diagnostic parasitology. Vet Clin North Am Small Anim Pract. 1989;19(5):979-1000.

27. Dryden MW, Payne PA, Ridley R, Smith V. Comparison of common fecal flotation techniques for the recovery of parasite eggs and oocysts. Vet Ther. 2005;6(1):15-28.

28. Tegzes JH, Puschner B. Amanita mushroom poisoning: Efficacy of aggressive treatment of two dogs. Vet Hum Toxicol. 2002;44(2):96-99.

29. Puschner B, Rose HH, Filigenzi MS. Diagnosis of Amanita toxicosis in a dog with acute hepatic necrosis. J Vet Diagn Invest. 2007;19(3): $312-317$.

30. Kauserud H, Heegaard E, Halvorsen R, Boddy L, Høiland K, Stenseth NC. Mushroom's spore size and time of fruiting are strongly related: is moisture important? Biol Lett. 2011;7(2):273-276.
31. Gausterer C, Penker M, Krisai-Greilhuber I, Stein C, Stimpfl T. Rapid genetic detection of ingested Amanita phalloides. Forensic Sci Int Genet. 2014;9:66-71.

32. Epis S, Matinato C, Gentili G, Varotto F, Bandi C, Sassera D. Molecular detection of poisonous mushrooms in different matrices. Mycologia. 2010;102(3):747-754.

33. Harper KA, Smart CD, Davis RM. Development of a DNA-based macroarray for the detection and identification of Amanita species. J Forensic Sci. 2011;56(4):1003-1009.

34. Maeta K, Ochi T, Tokimoto K, et al. Rapid species identification of cooked poisonous mushrooms by using real-time PCR. Appl Environ Microbiol. 2008;74(10):3306-3309.

35. Robinson-Fuentes VA, Jaime-Sánchez JL, García-Aguilar L, Gómez-Peralta M, Vázquez-Garcidueñas MS, Vázquez-Marrufo G. Determination of $\alpha$ - and $\beta$-amanitin in clinical urine samples by capillary zone electrophoresis. J Pharm Biomed Anal. 2008;47(4-5): 913-917.

36. Kaya E, Karahan S, Bayram R, Yaykasli KO, Colakoglu S, Saritas A. Matoxin and phallatoxin concentration in Amanita phalloides spores and tissues. Toxicol Ind Health. 2013:1-6. Epub May 29, 2013.

37. Fender SJ, Watling R. Effects of digestion on agaric structures. Edinburgh J Bot. 1991;48(1):101-106.

38. Brandin AC, Meola SD, Mazzaferro EM. Respiratory arrest following ingestion of wild mushrooms in 3 dogs (2006-2011). J Vet Emerg Crit Care (San Antonio). 2013;23(6):605-609.

39. Hall IR, Stephenson SL, Buchanan PK. Edible and Poisonous Mushrooms of the World. Portland, USA: Timber Press; 2003.
Veterinary Medicine: Research and Reports

\section{Publish your work in this journal}

Veterinary Medicine: Research and Reports is an international, peer-reviewed, open access journal publishing original research, case reports, editorials, reviews and commentaries on all areas of veterinary medicine. The manuscript management system is completely online and includes a very quick and fair peer-review system.

\section{Dovepress}

Visit http://www.dovepress.com/testimonials.php to read real quotes from published authors. 